\title{
APPLICATION OF MALARIA DETECTION OF DRAWING BLOOD CELLS USING MICROSCOPIC OpenCV
}

\author{
Antonius Herusutopo; Rahadi Wibowo Bisono; Josephta Inri Meliala \\ Computer Science Department, School of Computer Science, Bina Nusantara University \\ Jl. K. H. Syahdan No-9, Jakarta 11480, Indonesia \\ ant.herusutopo@binus.ac.id
}

\begin{abstract}
The goal of the research is to produce an application, which can detect malaria on patient through microscopic digital image of blood sample. The research methods are data collection, design analysis, testing and evaluation. The used application methods are image pre-processing, morphology and image segmentation using OpenCV. The expected result is a creation of application, which can be able to detect malaria on a microscopic digital image of patient blood sample. The conclusion is that the application can detect malaria from young trophozoites stadium and gametesocytes from the picture.
\end{abstract}

Keywords: Detection; Malaria; Computer Vision; OpenCV

\section{INTRODUCTION}

System technology of computer-based with artificial intelligence already can be used in medicine field, for example, to resolve the problems: detecting specific disease and its symptoms, analyzing the content of a sample, monitoring the condition of an organ, and others. Nevertheless, the medical field is very wide, so for detecting diseases problems, not yet much disease that detection can be done with a computer-based system. One example of the issues is well-known disease detection, which is malaria. Malaria is classified as a serious disease because it can cause death if it is not treated properly. Malaria has various types and can affect anyone anywhere. The symptoms of malaria is really common as it may appear in daily life, but cannot always indicate that a person infected with malaria. Indications, which can show that a person infected with malaria, are the clinical examination and blood tests.

With the blood test, the treatment of malaria can be implemented correctly and precisely. It needs technology that can detect malaria correctly and precisely. The solution is the method of support vector machine that can detect malaria in humans by viewing image of appearance blood cells.

\section{METHOD}

The methods used in this research are data collection, analysis and design. The data collection includes literature studies about computer division with OpenCV and data collection of microscopic of blood sample. Analysis method includes process, detection procedure and malaria diagnosis. While design method includes steps of detection implementation and diagnosis to the application program, coding and continued with evaluation.

\section{Malaria}

Malaria parasites in human have a life cycle that requires a human host and mosquito host. In the anopheles mosquito, plasmodium does sexual reproduction. In humans, these parasites asexual reproduction, starting in the liver cells (hepatocytes), then repeatedly in the red blood cells (erythrocytes).

While an infected female anopheles mosquito is sucking human's blood, at the same time the mosquito inserts its saliva that is to keep the capillary vessels, which is inhaled not forming a blood clots factor that causes the blood flow stops. At this time the parasite creates sporozoites to enter the blood flow and infect hepatocytes. For one until two weeks (depends on plasmodium species), each sporozoites creates schizont; a structure that contains thousands of merozoites. When schizont is mature, hepatocytes will rupture and release merozoites to blood flow.

In plasmodium vivax and plasmodium ovale, sporozoites develops into hipnozoit; a form of plasmodium that in dorman phase during several months to years. When hipnozoit re-activate, they will evolve into schizont that will cause recurrent symptoms to the infected person.

Next is the merozoites, which is released to the blood flow, will invade erythrocyte then they will grow and consume hemoglobin. In erythrocyte, half of merozoites will grow to another phase of asexual, which creates schizont filled with merozoites. When schizont is mature, the cell will rupture and merozoites will be released and invade erythrocyte. 
This infection will continue until terminated by the drug or the body's immune system.

Next is plasmodium will complete its life cycle when half of merozit on erythrocyte do not grow to schizont, but grow as male and female sexual form named gametesocytes. Gametesocytes will circulate in blood, waiting for anopheles mosquito to suck blood.
When female mosquito sucks gametesocytes from the infected person, gametesocytes will create male and female gametes. Fertilization process will create oocytes that filled with sporozoites. Then the oocytes are mature and rupture, thousands of infectious sporozoites will migrate to the mosquito's salivary glands. This cycle will repeat when the mosquito sucks blood from the next human.

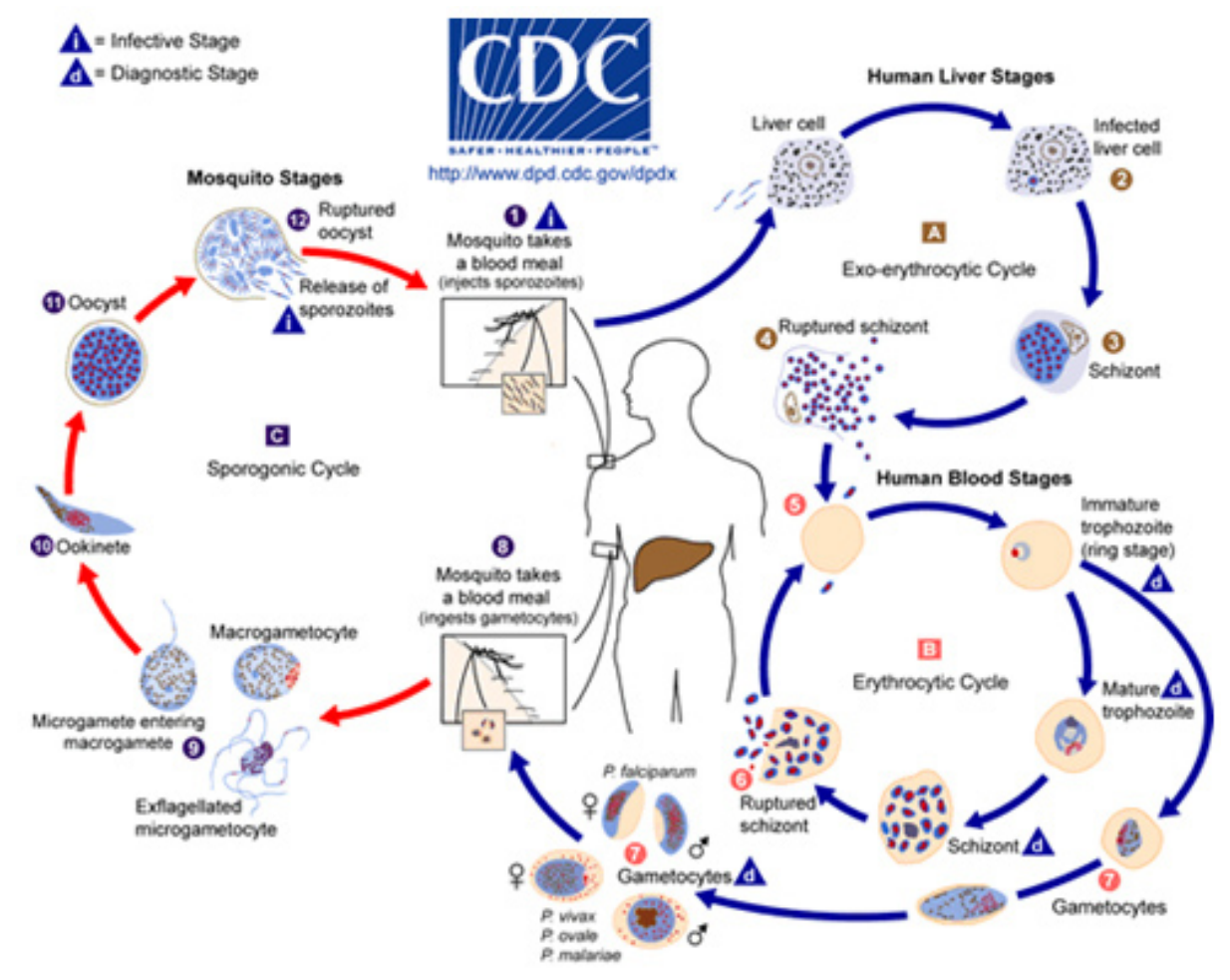

Fig 1 : Life Cycleof MalariaParasites

\section{Malaria Symptoms}

Typically, malaria creates intermittent attacks, or paroxysmal, every attack consists of three steps, which are trembling, follows with fever, then sweating. In trembling step, the patient will have headache, malaise, fatigue, muscle aches and sometimes nausea, puke and diarrhea. In one until two hours, body's temperature will increase; skin will be hot and dry. Then along with the decreasing body temperature, the patient will sweat a lot and feel tired and weak.

The above symptoms firstly appear 10-16 days from the infected mosquito sucks the blood. At the time the attacked erythrocyte ruptures together then the symptoms above appear. The cycle is different on every plasmodium species, so the duration of diseases is different on every species. The attacked symptoms appear every two days on plasmodium vivax malaria and plasmodium ovale, and every three days on plasmodium malaria.
The patient with plasmodium vivax malaria will feel healthy among the malaria attack, while in plasmodium falciparum malaria, the patient will keep feel sick because plasmodium falciparum can attack erythrocyte on any development phase, so the total of the infected erythrocyte become a lot than other malaria types. This can cause plasmodium falciparum malaria becomes the most fatal malaria. Plasmodium vivax malaria can be the more light symptoms because this parasite only attacks young erythrocyte that has few amount in blood flow, so the total of parasites in blood flow are not as much as on plasmodium falciparum malaria.

\section{Malaria Diagnosis}

The way to distinguish the type of malaria can be done on examination preparation thin and thick blood smear. In the preparation of thin blood, parasites can be found scattered and more difficult to find. Erythrocyte not undergo hemolysis (the 
breakdown of red blood cells), making them easier to identify stages and species of parasites.

Erythrocyte changes are as follows: (1) The attacked erythrocytes enlarge: Erythrocytes, which are attacked, are round shape, flat edges and can be found in all stages of the parasite (Tertian malaria caused by plasmodium vivax). If the shape of the attacked erythrocytes is oval, serrated edges (fimbrated) and containes all parasite stages (Tertian malaria caused by plasmodium ovale). (2) The attacked erythrocytes are not enlarged: Generally there are only young trophozoites or gametocytes stages shaped like a banana (Tropical Malaria caused by plasmodium falciparum). There are all stages of the parasites (Quartana malaria caused by plasmodium malariae).

In preparation of thick blood, it is found the parasite assemble and easier to search for. In this preparation also found erythrocytes that experience hemolysis, making it more difficult to identify the stage and parasite species so that the diagnosis is a disease or malaria parasite species diagnosis.

Patients with malaria provide an overview of blood preparation depends on the stage that is being experienced: (1) Overview of one stage: Generally there is only young trophozoite stage (or with gametocytes). Since the number of young trophozoite stage contained in the preparation, it looks like a picture of the stars in the sky with its gametocytes in banana-shaped or crescent (tropical malaria caused by plasmodium falciparum). (2) Description of various stage: The presence of amuboid (the continues of trophozoite) with a specific red zone. Shape and a more advanced stage are proportional to the magnitude of leukocyte core (tertian malaria caused by plasmodium vivax). The presence of typical skizont (such as chrysanthemums) that the amount of one-third to one-half of the leukocytes core and coarse pigment (quartana malaria caused by plasmodium malariae). Tertian malaria caused by plasmodium ovale is still difficult to distinguish because the preparation results are similar to the plasmodium vivax and malariae.

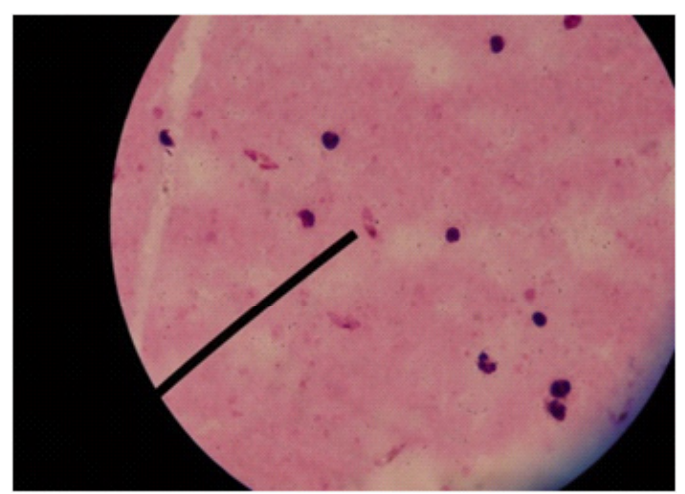

Fig. 2: Thic kBlood sample preparation of Plasmodium Falciparum
Some ways to distinguish the type of malaria from the thick blood clots picture are as follows: (1) Plasmodium Falciparum: The red blood cell has already experienced hemolysis. Young trophozoite looks a lot, with forms such as commas, exclamation point, birds fly so as to provide an overview as the stars in the sky (sterren hemel). Gametocytes look like banana, with a core and dispersed pigment. (2) Plasmodium Vivax: The red blood cell has already experienced hemolysis. There is the presence of the red zone, such as remnants schuffner point. The discovery of the young trophozoite stage is in ring form, trophozoite stage further amuboid, and schizont stage. (3) Plasmodium Malariae: Red blood cells have experienced hemolysis; it looks like trophozoite stage, gametocytes, schizont with an arranged core like flower. Parasite seems small when compared to other leukocytes core, with a rough brown pigment tengguli.

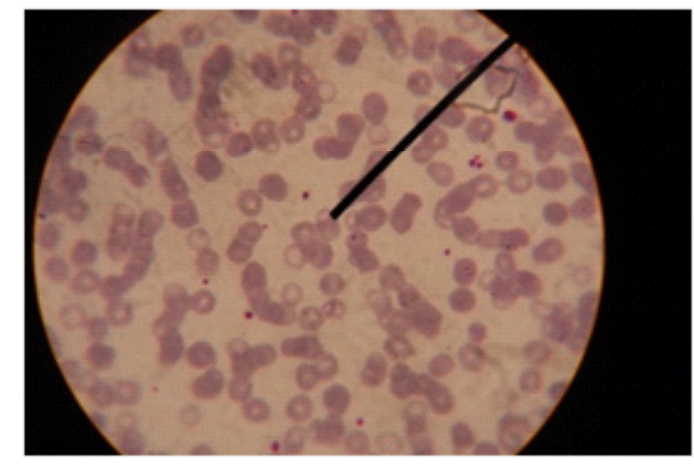

Fig. 3: First Sample of Plasmodium Falciparum Thin Blood Preparation

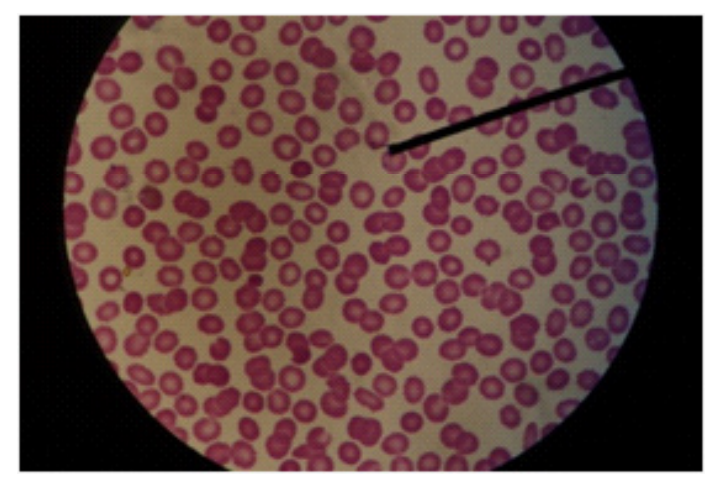

Fig. 4: First Sample of Plasmodium VivaxThin Blood Preparation

Next is some ways to distinguish the type of malaria on thin blood preparations image is as follows: (1) Plasmodium Falciparum of Young Trofozoit: Red blood cells are not enlarged, ring-shaped, there are often multiple infections and parasites are usually located on the edge of red blood cells. Sometimes it seems Maurer point depends on the coloring. (2) Plasmodium Vivax of Young Trofozoit. 
Red blood cells begin to expand, the ringshaped parasites. The core of red and blue cytoplasm and there is schuffner point.

\section{Computer Vision}

According to Ref. [1], computer vision is associated with capturing pictures, processing, classification, recognition and becomes the incorporation of decisions sequence towards recognition. And according to Ref. [2], computer vision is a field that aims to make useful decisions regarding the real physical objects and circumstances based on an image. Computer vision is a combination of image processing and pattern recognition, which seeks to enable the computer to recognize the object being observed. The output of the computer vision is the understanding about the image.

According to Ref. [3], computer vision can be interpreted as a transformation of the image data or video captured by the camera into a new representation, where the representation can be made into a decision-making. All transformations, which are performed in computer vision, aim to achieve a goal, namely to make useful decisions about an object based obtained or captured image by the hardware. Although viewing an image on computer just as numbers, but with computer vision we can build intelligent machines that can interpret the image as seen through different strategies depending on the condition.

\section{Computer Vision Hierarchy}

According to Ref. [4], several stages in computer vision include the acquisition of the image, preprocessing, feature extraction, associative storage, access the knowledge base and recognition. The stages are shown as a chart in Fig 5.

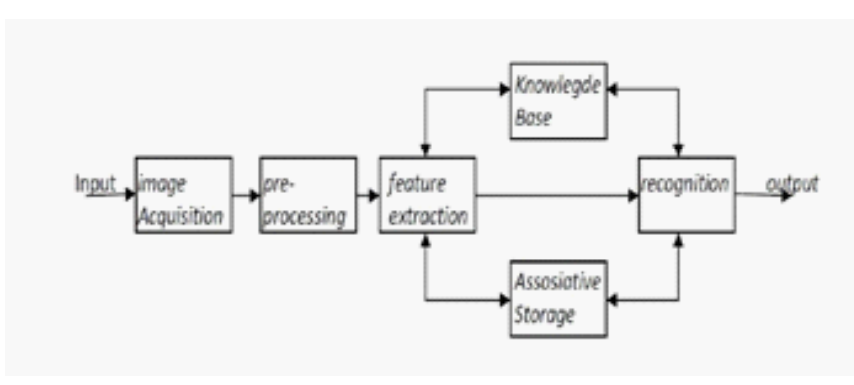

Fig. 5: System Chart of Computer Vision

According to Ref. [4], these stages can be classified into the following hierarchy levels: (1) Low-level vision: image enhancement and remove noise. (2) Intermediate-level vision: low-level vision accompanied by edge detection, texture detection and feature extraction. (3) High-level vision: lowlevel vision, intermediate-level vision, which is then followed by a phase of associative storage, knowledge base, and recognition.
The stages can be described as follows according to Ref. [5].

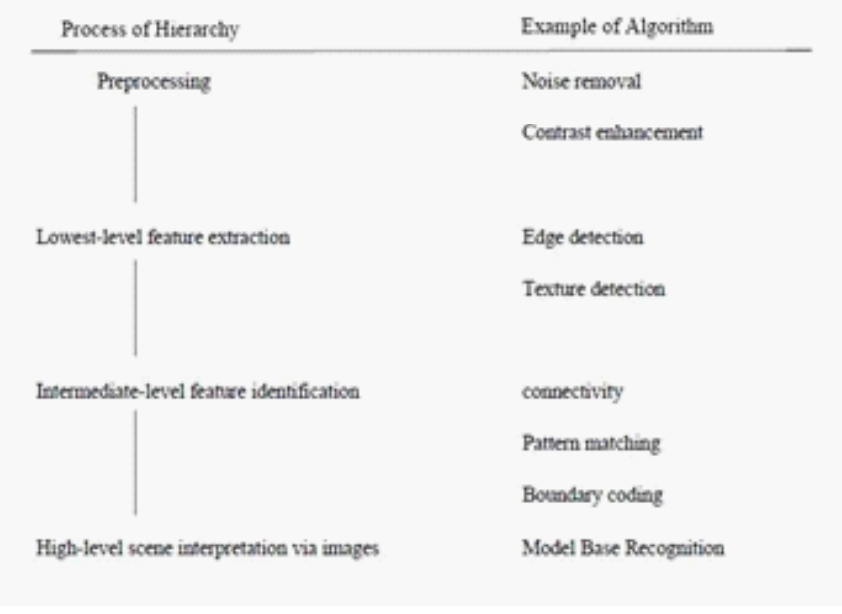

Fig. 6: Computer Vision Hierarchy

\section{Scope of Computer Vision}

According to Ref. [3], the most frequently encountered problem in computer vision is the amount of noise that interfere the image processing, feature extraction, and others. Tasks such as identifying signatures, recognize objects that are received from the image generated by the satellite, identify faces and build a three-dimensional image of a piece of two-dimensional image requires good image quality (less noise) so that processing can produce good decisions.

According to Ref. [6], the scope of computer vision includes some examples of applications as follows: (1) Optical Character Recognition (OCR): reading of handwriting and recognizing number plate automatically. (2) Machine inspection in the airline industry: checking the performance of aircraft parts. (3) Navigation: the auto-pilot, where the vehicles can run themselves to a particular destination. (4) Surveillance (monitoring): monitoring if there is an intruder or monitor traffic conditions. (5) Fingerprint recognition: fingerprint recognition for automated access. (6) Object modeling: medical image analysis or topographical models.

\section{Digital Image}

According to Ref. [2], digital image is used for a wide range of hardware and software that can read and use the images in the form of data. In daily life, there are various kinds of image data formats. Even so, the image data consists of specific information. The digital image has information in the form of images and consists of the smallest elements called pixels. Each element represents pixel in the image.

According to Ref. [5], the pixel is represented in the form of two-dimensional matrix and has value, which is a variation of the color components. All the 
existing color is a blend of three primary colors, which are: red, green and blue that is commonly referred to as RGB. The combination of the three primary colors will produce a certain color depending on the composition of the value of the three colors.

Besides that Ref. [5] also stated that, drawing on digital system can be represented by RGB format for every point. Each component of the R, G, and B has value variations from 0 to 255 . The total variation, which is generated for the digital color system, is 256 x 256 x 256 or 16,777,216 kinds of colors. Because each color is represented by one byte ( 8 bits), then the total of the total of bits, which is used to represent the color of RGB, is $8+8+8$ or 24 bits.

Image processing calculations with RGB system will waste memory and time. So that color reduction is needed. In the image processing such as object detection; RGB system is not providing a good response. Due to that the system uses gray scale or gray level, where the format of the color image is converted into gray image format. Gray scale system requires one byte ( 8 bits) for data storage, which has the possibility of color range from 0 (black) to 255 (white).

\section{Pre-Processing}

According to Ref. [4], pre-processing is a starting process to remove the parts that are not needed in the input image to the next process. Stage of pre-processing is usually done before we perform the processing core of the image. The purpose of the pre-processing are: (1) Eliminating noise. (2) Clarifying any data feature. (3) Enlarging or reduce the size of data. (4) Convert the original data in order to obtain data as needed.

Some examples of pre-processing are: (1) Noise filtering. (2) Conversion of RGB color to gray scale. (3) Thresholding.

\section{Smoothing}

Smoothing is a technique that is commonly used in image processing. This process aims to refine images, reduce image resolution and reduce noise [3].

The smoothing process uses convolution technique that uses kernel with various sizes. In the process, there are several ways to perform smoothing, such as the mean filter, median filter, Gaussian filter and bilateral filter, where in the simplest process of the smoothing result on a pixel is the result of the average pixel by pixel in the vicinity.

The basic concept is to find the value of the pixel, which has the intensity value of a pixel that is different from the pixel values in the surrounding area, and replaces it with a more suitable value. The simplest way to achieve it is by making prevention or restriction of pixel values, so that a pixel does not have a value beyond the value of the intensity around it [7].
For that we need to know the intensity value of a pixel group. In the process in a pixel area, which is supposed to be part of the highest and lowest values and the value, which is comparable in both parts of the final distribution, is eliminated. So the result leaves the median value. From that the median filter is obtained, where acquired the entire value of the intensity distribution and produced a new image that corresponds to the values of the existing median.

Unlike the gaussian filter, which refines the whole image, the median filter shows that the smoothing process occurs at the edges of the image. So despite the image smoothing, median filter more towards "soften" the image.

Median filter generally uses a kernel with $3 \times 3$ sizes but it can also use larger size. Furthermore, in accordance with its development, the form that is used can also vary, such as line (vertical or horizontal) or cross, which centered on the midpoint. It is intended that the resulting process becomes faster, mainly because the number of pixels, which is less calculated.

Median filter is quite well known for its ability to remove salt and paper noise. In addition, the median filter will improve the image quality, making clear edge region on the image [8]. This happens because the region is located at the edge of an image; the filter will process the data and will generally get the suitable value with the greater intensity value. So that the filter does not directly determine which parts the pixel exists. This surely makes the edge of the area becomes a bit wider. But keep in mind that the pixel widens the edge area of both sides, so it caused a strip edges can be seen [7].

\section{Image Segmentation}

In computer vision, image segmentation means the process of dividing the digital image into many segments. According to Ref. [5], the purpose of segmentation is to simplify and/or change the representation of the image into something more meaningful and easier to analyze. Image segmentation is usually used to locate objects and boundaries in the image field.

Some segmentation methods are as follows: (1) Thresholding Method: Thresholding or binerization is the process of converting the gray image into a black and white image. This process is also called (image binarization) and a simple method for image segmentation. This process is done by using a threshold value as limiting the complexity of the image. Thresholding process is used to convert the pixel values depend on the size of the pixel value of the predetermined threshold value. If the value of a pixel is greater than or equal to the threshold value, then the pixel will be set to the maximum value of the gray scale, which is 255 (white). Meanwhile, if the value of a pixel is less than the threshold value, 
then the pixel will be set to the minimum value in the gray scale is 0 (black). This process helps eliminate noise in the image. No provision limits about how much a threshold value. This threshold value can be changed as needed so that the conversion process produces the wished image. (2) Histogram-Based Method: Histogram-based method is more efficient than other methods of image segmentation because it usually requires one pass through the pixel. In this technique, histogram is calculated from all pixels in the image. Peaks and valleys in the histogram are used to find clusters in the image. The development of this technique is recursively perform a searching method in the image histogram to divide them into smaller clusters until no more clusters are made. (3) Distance Transform Method: Distance transform, which is also known as distances map, is a representation of a digital image. The term of map depends on the object, whether the initial image transformed into another representation, or simply given additional. Each pixel image is labeled with limiting distance to the nearest pixel. The most common type of pixel barrier is the boundary pixel in the binary image. Usually transformation based on the distance of the selected function. The results of the transformation is a gray scale image that looks similar to the initial image, except the intensity of the gray-level at the point in the foreground changed to indicate the distance to the nearest boundary of each point. (4) Watershed Segmentation Method: A gray scale image visualization can be represented as topography, where the gray value of a pixel is defined as the height of the relief. Watershed is a hollow form (dot) formed by the high points and Ridgeline (watershed line), which decreased to the lower points. Different approaches can be used for using the watershed for image segmentation. The first is the value of local minima of the gradient image as a marker; in this case segmentation is created, which then will involve the merging region. The second is markers watershed transformation based on specific position markers that have been explicitly defined by the user or automatically determined by morphological operators or other ways. The three basic elements in the watershed segmentation are minima values, the value of the basin and watershed lines. The purpose of watershed segmentation is to find all the watershed line (the highest gray level).

\section{OpenCV}

According to Ref. [3], OpenCV (Open Computer Vision) is an open source library that specialized from image processing-based $\mathrm{C} / \mathrm{C}++$, which is currently used in computer vision program. OpenCV can be obtained from "http://opencv.org/". The library is made with $\mathrm{C}$ language and $\mathrm{C}++$, and can also run in operating system like Windows, Linux, and Mac OS X.
One of the OpenCV goals is a computer that has the ability similar to the way of human visual processing. The advantage of OpenCV is a computer vision OpenCV provides the infrastructure that is easy to use, so as to assist the user quickly in building applications based on computer vision. OpenCV is designed for computational efficiency and with focus on real time applications. According to Ref. [9], the OpenCV Library contains over 500 functions that span a variety of computer vision problem areas such as, image processing, camera calibration, user interface and robotics. OpenCV also has a Machine Learning Library (MLL). OpenCV library consists of computer vision functions and APIs (Application Programming Interface) for image processing in a low-level and high-level vision.

According to Ref. [9], since the first launch in January 1999, OpenCV has been used in many applications, products, and research. These applications include the incorporation of images on the web and satellite, image scan alignment, image noise reduction in medical, security systems and interference detection, automatic surveillance and security systems, factory inspection system, fingerprint recognition, face recognition, as well as military applications. OpenCV has also been used for speech recognition, where the vision recognition techniques applied to the image of the sound spectrograms [3].

\section{OpenCV Features}

There are some of the features on the OpenCV library: (1) Manipulation of the image data (memory allocation, release the memory, duplicating the image, image conversion). (2) Image/ video I/O (input format / output in image or video that is connected to the hardware like the camera). (3) Manipulation of vector and matrix and linear algebra formulas (products, solvers, eigenvalues). (4) Image processing (filtering, edge detection, sampling and interpolation, color conversion, histogram). (5) Structural Analysis. (6) Detection and object recognition. (7) Image labeling (line, Conic, polygons, text drawing). (8) Basic GUI (display output, control input).

\section{RESULTS AND DISCUSSION}

The designed application system is an application that can detect malaria by using computer vision methods that exist in OpenCV 2.3 library. Core detection of malaria in this application is to detect the presence of young trophozoite stage (ring-form) and gametocytes in thin blood preparations microscopic images.

The application that we designed only has GUI with one screen. On the screen there are 4 menus which have each function. Then the console screen displays the status of malaria detection results. Moreover beside the screen, it will display a screen 
that contains image that has not and that has passed through the processing results of the detection of malaria.

In the first button is the 'Load Image', which serves to locate and retrieve the images that will be processed. Processed image file extension is *.png. Furthermore, the second button is the 'Malaria Detection', which serves to carry out the process of malaria detection in the image that has been loaded previously. In the processing, the entire of computer vision methods for the malaria detection run from start to finish. The method is image enhancement, parasite detection, segmentation red blood cell, red blood cell counting, counting parasites, abnormal red blood cell counting, and malaria detection.

After the processing, the image of malaria detection will be displayed on the image screen and the detection status information is displayed on the console screen that has emerged from the beginning of the run program. Then on the main screen there is a button 'Show Original Image', which serves to bring back the original picture image that has been loaded before. The aim of the 'Show Original Image' is to see the difference image before and after processing. Moreover, on the first screen there is a button 'Show Processed Image', which has the same goal with 'Show Original Image', but the displayed image is an image that has been processed.

After the detection of malaria, there are three information that are displayed on the console screen, which are the 'Infected by Malaria', 'Parasites Detected' and 'Normal Erythrocytes'. Besides that, it will appear different results of detection depending on the loaded image. The first displayed information is whether the detectable images contained malaria infection. If so it would appear 'Yes' in the 'Infected by Malaria', otherwise it will display 'No'. Second, the displayed information is the number of parasites detected in the 'Detected Parasites'. And the third is the amount of normal erythrocytes that are detected in the 'Normal Erythrocytes'.

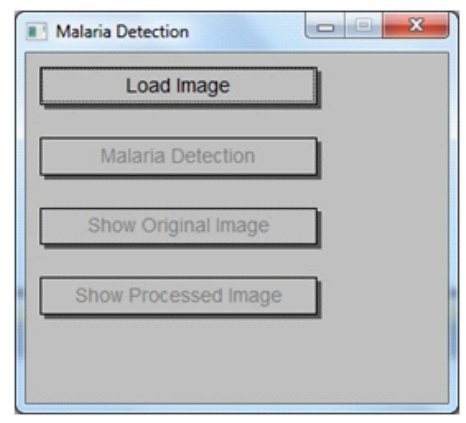

Fig. 7: Display application before the loaded image

Fig. 7 is the initial view when the application is run. In the Fig. 7 above, the application has not yet displayed images (blank) as well as the information is still not available, because the image to be processed is not yet loaded. Next to load the image that will be processed, the 'Load Image' button will be pressed. Then select the image to be processed. Once the image is selected, the image will appear on a separate screen (screen image).

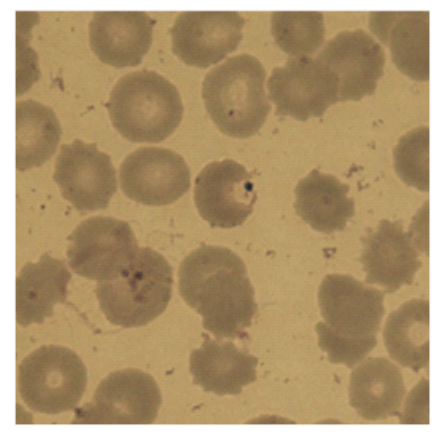

Fig. 8: Displayapplicationafter the loaded image

Fig 8 is a screen image that is separate from the main screen after loading the processed image. While on the main screen, nothing has changed and the information is still not there because the image has not been processed.

To make the process of malaria detection, the 'Malaria Detection' will be suppressed. Once pressed, the entire process of detection of image enhancement to malaria, parasites, normal erythrocytes detection is executed. The end result is a contour marking with a different color on each object in the loaded image.

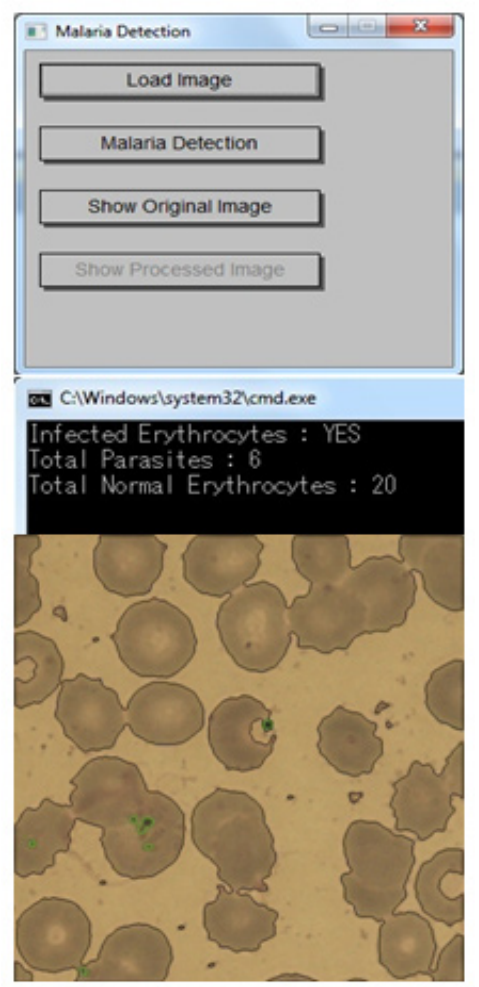

Fig. 9: Display after pressed 'Malaria Detection' Button 
Fig. 9 is the result after pressing the 'Malaria Detection'. In Fig. 9 can be seen that the image has been marked. Objects, which are marked with black lines, are red blood cells. While marked in green are parasites. Then it can also be seen on the console screen, the detection information results already displayed. To see the initial image before the detection, then press 'Show Original Image'. The image screen will display the initial image before processing, but the information on the display console will not be lost. To recall the image processing results, then press the button 'Show Processed Image'. The screen will display the processing results image. To load a new image, then press the "Load Image" and select the next image to do the detection process.

\section{Implementation and Evaluation Results}

There are the implementation results of Eijkman Institute for Molecular Biology data:
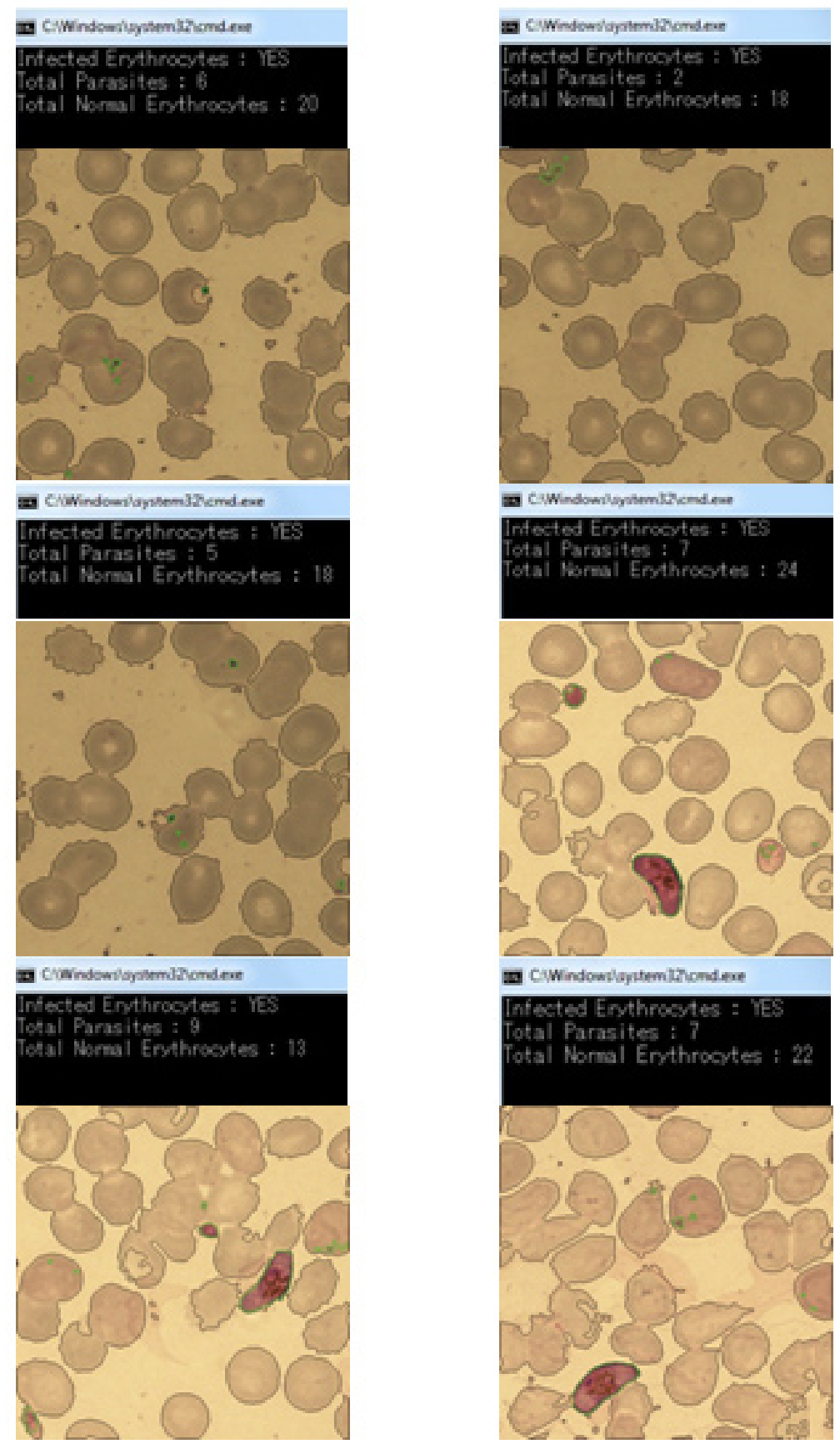

Fig. 10:Implementation of EijkmanInstitute Data for Molecular Biology 
Based on the implementation data of the above, the application can distinguish red blood cells and the parasite. This application can also calculate the detected number of parasites and red blood cells. However, based on the above data, the calculation of normal red blood cells is still not perfect because some implementation results show that the numbers do not fit in the calculation of normal red blood cells. The calculation becomes incompatible because the shape of red blood cells are not perfect (no full round) and red blood cells are fused with other red blood cells.

\section{CONCLUSION}

The conclusions that can be drawn from the results of the application design that have been made are: (1) This application can detect the presence of malaria in blood samples from blood preparations microscopic images, where the core detection seen from the young trophozoite (ring-form) stage and gametocytes in thin blood preparations microscopic images. (2) This application can detect malaria infection in samples of blood clots, where the condition of the red blood cells has a shape that is fully evident. (3) This application can perform the detection of malaria parasites and the number of red blood cells is normal (uninfected).

Nevertheless the known weaknesses of this application are: (1) The detection is difficult to be done and has an error if the blood sample images are much noise (dirty) and the picture quality is not good (too small dimensions and resolution). (2) Having error detection if the microscopic images are clumps of white blood cells that have no fixed shape or have a form such as the malaria parasite. (3) Error also occur in the calculation of the red blood cells numbers if the shape of the red blood cells are not perfect or blend with other red blood cells, so it is considered as a red blood cell.

\section{REFERENCES}

[1] A. Low, Introductory Computer Vision and Image Processing. Mcgraw Hill Book Co Ltd, 1991.

[2] L.G. Saphiro, G. Stockman, Computer Vision. USA: Prentice Hall PTR Upper Saddle River, NJ, 2001.

[3] G. Bradski, \& A. Kaehler, Learning OpenCV: Computer Vision with the OpenCV Library. O'Reilly Media, Inc, 2008.

[4] A. D. Kulkarni, Computer Vision and FuzzyNeural Systems. New Jersey: Prentice-Hall Inc, 2001.

[5] W. William, R. Mulya, A. Maharani, Analisis Dan Perancangan Sistem Pengenal Karakter Tulis Tangan Online Dengan Pendekatan Support Vector Machine. Undergraduate thesis,
BINUS, 2009.

[6] R. Szeliski, Computer Vision, Algorithms and Application, Texts in Computer Science. New York: Springer, 2011.

[7] E. R. Davies, Machine Vision. Academic Press, New York, 1990.

[8] M. Nixon, A. Aguado, Feature Extraction \& Image Processing. USA: Newnes, 2002.

[9] D. Chandra, N.Prajnagaja, A. L. Nugroho, Studi Pendeteksian Wajah dengan Metode Viola Jones. Undergraduate thesis BINUS, 2011. 